\title{
On orienting graphs for connectivity: projective planes and Halin graphs
}

\author{
Joseph Cheriyan \\ C\&O Dept., U. Waterloo * \\ Chenglong Zou \\ Mathematics Department, U. British Columbia,
}

\begin{abstract}
Nash-Williams proved that the edges of a $k$-edge connected (undirected) graph can be oriented such that the resulting directed graph is $\left\lfloor\frac{k}{2}\right\rfloor$-edge connected. A long-standing goal in the area is to obtain analogous results for other types of connectivity, such as node connectivity, element connectivity, and hypergraph edge connectivity. We focus on two special classes of graphs, namely, incidence graphs of projective planes and (generalized) Halin graphs, and we prove some analogs of Nash-Williams' result for these classes.
\end{abstract}

Keywords: Graph connectivity, Graph orientations, Element connectivity, Hypergraphs, Projective planes, Halin graphs.

\section{Introduction}

A well-known result of Nash-Williams says that the edges of a $k$-edge connected (undirected) graph can be oriented such that the resulting directed graph is $\left\lfloor\frac{k}{2}\right\rfloor$-edge connected $[13,2,7]$. This result is now more than 50 years old, and there are several proofs known, e.g., using the splitting-off technique, using submodular flows, etc.

A long-standing goal in the area is to extend Nash-Williams' result to obtain analogous results for other types of connectivity, such as node connectivity, element connectivity, and the edge connectivity of hypergraphs. More precisely, there are a number of well-known conjectures in this area.

\subsection{Conjectures related to Nash-Williams' Orientation Theorem}

Thomassen [15] (1989) proposed a conjecture.

For all $k$, there exists a value $f(k)$ such that all $f(k)$-connected graphs have a $k$-node connected orientation.

\footnotetext{
*(jcheriyan@uwaterloo.ca) Department of Combinatorics and Optimization, University of Waterloo, 200 University Ave. West, Waterloo, N2L 3G1, Ontario, Canada.
} 
Frank [6] (1995) proposed a sharp version of this conjecture.

Let $G=(V, E)$ be an undirected graph with $|V|>k$. Then $G$ has a k-node connected orientation if and only if for all $S \subseteq V$ with $|S|<k, \quad G-S$ is $2(k-|S|)$-edge connected.

Observe that Frank's conjecture implies that Thomassen's conjecture is valid with $f(k)=2 k$. Jordán has proved that $f(2) \leq 18$, thus settling the special case of Thomassen's conjecture for $k=2$, see [10], and also see $[1,11]$.

Recently, Kiraly and Lau [12] formulated a sharp conjecture for the notion of element connectivity of graphs: consider a graph $G=(V, E)$ whose node set is partitioned into a set of terminals, $Y \subseteq V$, and nonterminals (also called Steiner nodes); assume $|Y| \geq 2$. By an element we mean a nonterminal or an edge. The element connectivity of $G$ is the minimum number of elements whose deletion results in a graph that has at least two connected components that each have a terminal; this number equals the minimum over all pairs of terminals $s, t$ of the maximum number of $s, t$-paths that are disjoint on the elements.

Kiraly and Lau [12] asked whether a $k$-element connected graph has an orientation that is strongly $\left\lfloor\frac{k}{2}\right\rfloor$-element connected. (The strong element connectivity of a directed graph is given by the smallest number of elements whose deletion results in a directed graph such that no strongly connected component contains all of the terminals.)

Remark: Given a graph of node connectivity $k$, we can obtain a graph of element connectivity $\geq k$ by choosing any set of two or more nodes to be the set of terminals.

Now, consider a hypergraph $H$ and its bipartite representation $B(H)$, where $V(H)$ forms one part of the node bipartition of $B(H)$ (one color class), and $E(H)$ forms the other part. (For ease of exposition, we may use $B$ to denote $B(H)$; also, we may refer to a member of $E(H)$ as either an edge or a hyperedge, and we use the term edge connectivity rather than hyperedge connectivity for a hypergraph.) We take the set of terminals of $B$ to be $V(H)$, and the set of nonterminals of $B$ to be $V(B)-V(H)$. It can be seen that the element connectivity of $B$ corresponds to the edge connectivity of $H$, that is, the minimum number of hyperedges whose deletion results in a disconnected hypergraph. Thus, the orientation conjecture for element connectivity would imply that the hyperedges of a $k$-edge connected hypergraph can be oriented such that the resulting directed hypergraph is strongly $\left\lfloor\frac{k}{2}\right\rfloor$-edge connected. (An orientation of a hyperedge $e=\left\{v_{1}, \ldots, v_{i}\right\}$ means that one or more (but not all) of its incident nodes are designated as tails, and the rest (at least one) are designated as heads.) We mention that this conjecture holds for the special case of 3-uniform hypergraphs; this follows from Nash-Williams' strong orientation theorem applied to the bipartite representation $B$, and the fact that $B$ has $\ell$ edge disjoint paths between a pair of terminals $s, t$ iff it has $\ell$ element disjoint $s, t$-paths (since every nonterminal has degree $\leq 3$ ). 


\subsection{Smooth orientations}

An orientation of a graph $G$ is called smooth if the absolute value of the difference between the indegree and the outdegree of every node is at most one, that is, $\left|d^{\text {in }}(v)-d^{\text {out }}(v)\right| \leq 1, \forall v \in V(G)$. If the graph $G$ is Eulerian, then a smooth orientation satisfies $d^{i n}(v)=d^{o u t}(v), \forall v \in V(G)$; moreover, it can be seen that for every subset of the nodes $W$, the number of arcs leaving $W$ is equal to the number of arcs entering $W$. Therefore, every smooth orientation of a $k$-edge connected Eulerian graph results in a directed graph that is $\left\lfloor\frac{k}{2}\right\rfloor$-edge connected. A smooth orientation of an Eulerian graph can be found by orienting the edges of each connected component according to an Euler tour.

\subsection{Our results}

To the best of our knowledge, Frank's conjecture, as well as the orientation conjecture for element connectivity (and the specialization for hypergraph edge connectivity), are beyond the reach of current knowledge; it is possible that there exist counter-examples to some of these sharp conjectures. Our goal is to focus on some families of graphs that contain graphs of connectivity $k$ for all (sufficiently large) $k$, and to study some of these sharp conjectures for these families.

We focus on two families of graphs, (i) the incidence graphs of (combinatorial) projective planes, and (ii) the (generalized) Halin graphs.

The incidence graphs of projective planes are $d$-regular bipartite graphs. We study a special case of the orientation conjecture for element connectivity on these graphs. Whenever we consider a bipartite graph $B$ in the context of element connectivity, then we assume that one part of the node bipartition of $B$ (one color class) is the set of terminals $Y$, and the other part (other color class) is the set of nonterminals $X$; moreover, we denote $B$ by $B=(X, Y, E)$. Thus, our focus is on the orientation conjecture for hypergraph edge connectivity. We show that there exists a smooth orientation (of the incidence graphs of projective planes) that has the required strong element connectivity. It may be possible to extend these results to other families of hypergraphs of interest in algebraic graph theory [8]; perhaps, some new general techniques could be developed.

A (generalized) Halin graph of node connectivity $k \geq 3$ is obtained by starting with a tree $T$ such that every internal node has degree $\geq k$, then adding a cycle $C$ on the leaves, and then adding $k-3$ copies of $T$ that are disjoint on the internal nodes of $T$ but have the leaf nodes in common, see [9]. Let us denote the resulting graph by $H G(k, T)$. For example, we may take $k=3$, and $T=K_{1, n}$ (a star); then we see that $H G(3, T)$ is the wheel graph with $n+1$ nodes.

Given an orientation of an edge $e=\{v, w\}$, we use the term opposite orientation of $e$ to mean the orientation where the tail and the head are swapped. 
Consider an orientation of $H G(k, T)$ where the edges of the cycle $C$ are oriented consistently (say, all clockwise); moreover, consider any smooth orientation $\widehat{T}$ of $T$; half of the copies of $T$ (including

$T$ ) get the same orientation as $\widehat{T}$, and the remaining copies of $T$ get the opposite orientation. (For an integer $j \geq 0$, we use the term half of $j$ to mean $\left\lfloor\frac{j}{2}\right\rfloor$.) We show that the resulting oriented graph has the required node connectivity; thus, Frank's conjecture holds for these graphs.

Our results emphasize an insight of Nash-Williams from more than 50 years ago: some smooth orientations have surprisingly good properties.

\section{Preliminaries}

This section has some definitions, and a preliminary result on orienting a subclass of 4-element connected bipartite graphs; the proof of this result illustrates a key tool for our result on the incidence graphs of projective planes.

For a bipartite graph $B=(X, Y, E)$ and a nonempty, proper subset $U$ of the terminals $Y$, we use $\Phi(U) \subseteq X$ to denote the set of those nonterminals that have a neighbor in $U$ and a neighbor in $Y-U$, and we call the nonterminals in $\Phi(U)$ the portals of $U$. Given an orientation of $B$ and a set of terminals $U, \emptyset \neq U \subset Y$, we call $x \in \Phi(U) \operatorname{good}$ (with respect to $U$ ) if one of the edges between $U$ and $x$ is directed into $x$ and one of the edges between $Y-U$ and $x$ is directed out of $x$; a nonterminal in $\Phi(U)$ is called bad (w.r.t. $U$ ) if it is not good. A key observation is that a bad portal $x$ either

(i) has all edges between $U$ and $x$ directed out of $x$, or

(ii) has all edges between $Y-U$ and $x$ directed into $x$.

Given an orientation of $B$ and a set of terminals $U, \emptyset \neq U \subset Y$, let $\Phi^{\text {out }}(U) \subseteq \Phi(U)$ denote the set of good portals (w.r.t. $U$ ); similarly, define $\Phi^{i n}(U)$ to be the set of portals $v$ such that there exists a dipath of length two from $Y-U$ to $U$ that contains $v$.

For subsets $U, W$ of nodes we use $\delta(U, W)$ to denote the set of (undirected) edges with one end in $U$ and one end in $W$; if $U$ or $W$ is a singleton set, say $\{v\}$, then we simplify the notation and use $\delta(v, W)$ or $\delta(U, v)$. Similarly, for an oriented (or, directed) graph, we use $\widehat{\delta}(U, W)$ to denote the set of arcs with tails in $U$ and heads in $W$.

Proposition 1 Let $B=(X, Y, E)$ be an Eulerian 4-element connected bipartite graph such that every nonterminal node (node in $X$ ) has degree 4 . Let $\widehat{B}$ be any smooth orientation of $B$. Then $\widehat{B}$ is strongly 2-element connected. 
Proof: The proof is by contradiction. Suppose the result fails to hold. Then, it can be seen that there exists a nonempty, proper subset $U$ of $Y$ such that $\Phi^{\text {out }}(U)$ has size $\leq 1$, that is, $U$ has $\leq 1$ good portal and has $\geq 3$ bad portals. Now, consider the set $W$ of nodes obtained from $Y-U$ by adding every bad portal of $U$ of type (i), and every nonterminal $x \in X$ that is not adjacent to $U$; in other words, $W=(Y-U) \bigcup\{x \in \Phi(U): \widehat{\delta}(U, x)=\emptyset\} \bigcup\{x \in X: \delta(U, x)=\emptyset\}$. Clearly, the number of arcs leaving $W$ is equal to the number of arcs entering $W$. Each bad portal of $U$ contributes at least one arc leaving $W$, hence, there are $\geq 3$ arcs leaving $W$. Each of the arcs entering $W$ must have a good portal of $U$ as a tail, hence, there are $\leq 2$ arcs entering $W$ (since there is $\leq 1$ good portal, and each portal has outdegree 2 in a smooth orientation). This gives the desired contradiction.

\section{Incidence graphs of projective planes}

This section focuses on the incidence graphs of projective planes. These are regular bipartite graphs, and the node connectivity equals the degree. If we take the set of terminals to be one set of the node bipartition, then the element connectivity equals the degree. We show that a smooth orientation of the bipartite graph has the required strong element connectivity (of half the degree).

A combinatorial projective plane is a set of points and lines, together with an incidence relation between points and lines that satisfies the following properties:

1. Given any two distinct points, there is exactly one line incident with both of them.

2. Given any two distinct lines, there is exactly one point incident with both of them.

3. There exist four points such that no line is incident with more than two of them. (This is used to exclude some degenerate cases.)

We denote the unique line incident to points $p$ and $q$ by $\ell_{p, q}$. A projective plane has an associated positive integer $N \geq 2$ called the order of the plane; the number of points is $N^{2}+N+1$, the number of lines is $N^{2}+N+1$, each point is incident to $N+1$ lines, and each line is incident to $N+1$ points. (For each prime power $p^{j}$, there exists a projective plane of order $p^{j}$.) Let $B$ be the bipartite graph that has a node for each point, a node for each line, and an edge between (the node of) a point and (the node of) a line iff the point and the line are incident in the projective plane. We refer to the nodes of $B$ as points or lines. Thus $B$ is the incidence graph of the projective plane. Clearly, $B$ is a $d$-regular graph with $d=N+1$. When discussing the element connectivity of $B$, we take the points of $B$ to be the terminals; thus, the lines of $B$ are the nonterminals.

The next result is well known, see [3], for example. 
Proposition 2 Let $B$ be the incidence graph of a projective plane of order $N$. Then $B$ has node connectivity $d=N+1$.

It follows from the previous result that the element connectivity of $B$ (with the points as terminals) is equal to the degree $d$.

The main result of this section shows that the orientation conjecture for element connectivity holds for the incidence graphs of projective planes. If the order $N$ is odd, then the incidence graph is Eulerian, and any smooth orientation suffices. Otherwise, we obtain a suitable orientation by removing (the edge set of) a perfect matching, choosing any smooth orientation of the remaining Eulerian graph, and choosing arbitrary orientations for the edges of the perfect matching; recall that a $d$-regular bipartite graph with $d \geq 1$ has a perfect matching, see [2].

Theorem 3 Let $B=(X, Y, E)$ be the incidence graph of a projective plane of order $N \geq 2$, let $d=N+1$ denote the degree, and let each point be a terminal. If $d$ is even, then let $\widehat{B}$ be any smooth orientation of $B$. Otherwise, let $\widehat{B}$ be the smooth orientation that results by first removing a perfect matching $M$ from $B$, then fixing a smooth orientation of the remaining graph $B-M=(X, Y, E-M)$, and then fixing arbitrary orientations for the edges of $M$. The strong element connectivity of $\widehat{B}$ is $\left\lfloor\frac{d}{2}\right\rfloor$.

Proof: The proof is by contradiction, and builds on the proof of Proposition 1.

Let $k$ denote $\left\lfloor\frac{d}{2}\right\rfloor=\left\lfloor\frac{N+1}{2}\right\rfloor$, the required strong element connectivity. Thus $k=\frac{N+1}{2}$ if $N$ is odd, and $k=\frac{N}{2}$ if $N$ is even. If $d=N+1$ is even, then we focus on the graph $B$ and the oriented graph $\widetilde{B}=\widehat{B}$. Otherwise ( $d$ is odd), we focus on the graph $B-M$ and the oriented graph $\widetilde{B}=\widehat{B-M}$. Thus, if $d$ is odd, then we focus on the graph obtained from $B$ by removing the perfect matching $M$, and on any smooth orientation of this graph; all of our terms (adjacency, neighbor, portal, etc.) are w.r.t. $B-M$ rather than $B$. In either case, the unoriented version of $\widetilde{B}$ is regular; let $\operatorname{deg}(\widetilde{B})$ denote the degree of any node; observe that $\operatorname{deg}(\widetilde{B})=2 k$, and moreover, $\widetilde{B}$ has $d^{\text {in }}(v)=k=d^{\text {out }}(v), \forall v \in V(B)$.

Suppose the result fails to hold. Then, it can be seen that there exists a nonempty, proper subset $U$ of $Y$ such that $\Phi^{\text {out }}(U)$ has size $\leq k-1$, that is, $U$ has $\leq k-1$ good portals.

First, observe that $|U| \geq k$. To see this, assume that $|U|<k$; consider any point $u \in U$ and $k$ of its out-neighbors $x_{1}, \ldots, x_{k}$ (these exist since $d^{o u t}(u)=k$ ); each of these nonterminals $x_{i}$ has $d^{\text {out }}\left(x_{i}\right)=k$, hence, it has an out-neighbor in $Y-U$; thus, each $x_{i}$ is a good portal of $U$, so $\Phi^{\text {out }}(U)$ has size $\geq k$. In other words, if $\left|\Phi^{\text {out }}(U)\right|<k$, then $|U| \geq k$. Similarly, it can be seen that $|Y-U| \geq k$. 
Let $q$ denote $|X-\Phi(U)|$, the number of Steiner nodes that are not portals. Since the number of good portals is $\leq k-1$, the number of bad portals is $\geq|X|-(k-1)-q$.

Using the same argument as in the proof of Proposition 1, we consider the set $W=(Y-$ $U) \bigcup\{x \in \Phi(U): \widehat{\delta}(U, x)=\emptyset\} \bigcup\{x \in X: \delta(U, x)=\emptyset\}$. Each bad portal of $U$ contributes at least one arc leaving $W$, each good portal of $U$ contributes $\leq k \operatorname{arcs}$ entering $W$, and there are no other arcs entering $W$. This gives $\geq|X|-(k-1)-q$ arcs leaving $W$, and $\leq k(k-1)$ arcs entering $W$. The number of arcs leaving $W$ is equal to the number of $\operatorname{arcs}$ entering $W, \operatorname{since} \operatorname{deg}(\widetilde{B})$ is even and the orientation is smooth. Hence, we have $k(k-1) \geq|X|-(k-1)-q$, or $q \geq|X|-\left(k^{2}-1\right)$.

For any set of terminals $Y^{\prime} \subseteq Y$, let $\Gamma\left(Y^{\prime}\right)$ denote the set of nonterminals adjacent to $Y^{\prime}$ (both portals and non-portals).

We have two cases; either (i) $\Gamma(U)=X$ or $\Gamma(Y-U)=X$, or (ii) $\Gamma(U) \neq X$ and $\Gamma(Y-U) \neq X$.

Consider the first case. We focus on the subcase where $\Gamma(U)=X$; the other subcase $(\Gamma(Y-U)=$ $X)$ is similar and we omit the details. Observe that we always have $q=2|X|-|\Gamma(U)|-|\Gamma(Y-U)|$. Combining this with our lower bound on $q$, we have $q=|X|-|\Gamma(Y-U)| \geq|X|-\left(k^{2}-1\right)$, hence, $k^{2}-1 \geq|\Gamma(Y-U)|$. Moreover, $Y-U$ contains a subset $S$ of size $k$ and $\Gamma(S)$ has size $\geq|S| \operatorname{deg}(\widetilde{B})-\left(\begin{array}{c}|S| \\ 2\end{array}\right)=k(2 k)-\left(\begin{array}{c}k \\ 2\end{array}\right)=2 k^{2}-\frac{k(k-1)}{2}=k(3 k+1) / 2$, hence, we have $|\Gamma(Y-U)| \geq$ $k(3 k+1) / 2$. This gives a contradiction, namely, $k^{2}-1 \geq k(3 k+1) / 2$, or $0 \geq k^{2}+k+2$.

Now, consider the second case. In this case, there exist two nonterminals $x^{\prime}, x^{\prime \prime}$ such that all of the neighbors of $x^{\prime}$ are in $U$ and all of the neighbors of $x^{\prime \prime}$ are in $Y-U$. Then $d=N+1$ is odd, $k=\frac{N}{2}$, and $\widetilde{B}$ has been obtained from $B$ by removing a perfect matching. This holds because any two lines of a projective plane have a point in common; in other words, $x^{\prime}$ and $x^{\prime \prime}$ have a common neighbor in $B$; but $x^{\prime}$ and $x^{\prime \prime}$ have disjoint neighborhoods in (the unoriented version of) $\widetilde{B}$; in other words, $\widetilde{B}$ is an orientation of $B-M($ not of $B$ ), hence, the degree $d=N+1$ is odd. Next, we claim that $q \leq 4 N$. We have $|\Gamma(U)| \geq N^{2}-N+1$, because $x^{\prime}$ has $\operatorname{deg}(\widetilde{B})=N$ neighbors in $U$, each of these points has $\operatorname{deg}(\widetilde{B})=N$ neighbors in $X$, and $x^{\prime}$ is the only common neighbor (for any two of these $N$ points). Then the number of nonterminals that have all neighbors in $Y-U$ is $|X-\Gamma(U)| \leq|X|-\left(N^{2}-N+1\right)=2 N$. Similarly, the number of nonterminals that have all neighbors in $U$ is $|X-\Gamma(Y-U)| \leq|X|-\left(N^{2}-N+1\right)=2 N$. Hence, we have $q \leq 4 N$; our claim is proved. Combining this with our lower bound on $q$, we have $4 N \geq q \geq|X|-\left(k^{2}-1\right)=$ $\left(N^{2}+N+1\right)-\left(\frac{N^{2}}{4}-1\right)=\frac{3 N^{2}}{4}+N+2$. This implies, $3 N^{2}-12 N+8 \leq 0$. The quadratic has roots $2 \pm \frac{\sqrt{12}}{3} \approx 0.8452,3.1548$, hence, we have $N \leq 3$ (otherwise, the quadratic is positive). Recall that $d=N+1$ is odd, hence, we cannot have $N=3$. Thus we have $N=2$ and $d=3$. Then, it can be seen that $\widehat{B}$ is strongly connected; alternatively, Nash-Williams' theorem gives an orientation that is strongly connected (a 3-edge connected graph has an orientation that is strongly connected). 
Thus our assumption fails to hold; in other words, every nonempty, proper subset $U$ of the terminals has $\left|\Phi^{\text {out }}(U)\right| \geq k$; similarly, each such set $U$ has $\left|\Phi^{i n}(U)\right| \geq k$.

\section{Halin graphs}

This section has a proof of our claim that the specified smooth orientation of Halin graphs (given in Section 1.3) achieves the desired node connectivity.

Let $G$ be a directed graph. A set of nodes $S \subseteq V(G)$ is said to be $k$-node connected if there exist $k$ openly disjoint $v, w$-dipaths, for every ordered pair of nodes $v, w \in S, v \neq w$. A $k$-fan from a node $z$ of $G$ to a set of $k$ or more nodes $U \subseteq V(G)-\{z\}$ means a set of $k$ dipaths such that each of these dipaths starts at $z$ and ends at a node of $U$, and moreover, $z$ is the only node that occurs in two or more of these dipaths. Similarly, a reverse $k$-fan from $U$ to $z$ means a set of $k$ dipaths from $U$ to $z$ such that $z$ is the only node that occurs in two or more of these dipaths.

Our proof uses the following standard method for verifying that a given directed graph $G$ is $k$-node connected, see $[4,5]$. First, we pick a set $S^{*} \subseteq V(G)$ of $k$ or more nodes. Then we verify that $S^{*}$ is $k$-node connected. Finally, for each of the remaining nodes $z$, we verify that there exists a $k$-fan from $z$ to $S^{*}$ and there exists a reverse $k$-fan from $S^{*}$ to $z$. See Even [4] and Even and Tarjan [5] for a proof of correctness and further details.

Proposition 4 (Even) Let $G$ be a directed graph, and let $S^{*}$ be a set of nodes with $\left|S^{*}\right| \geq k$ such that $S^{*}$ is $k$-node connected. Moreover, for each node $z \in V(G)-S^{*}$, suppose that there exist a $k$-fan from $z$ to $S^{*}$ and a reverse $k$-fan from $S^{*}$ to $z$. Then $G$ is $k$-node connected.

Given an $\operatorname{arc} e=v w$ of a directed graph, we say that a node $x \neq v$ is reachable from $e$ if there exists a $v, x$-dipath that starts with the arc $e$. We also use the following simple fact.

Fact 5 Let $\widehat{F}$ be a smooth orientation of a tree $F$, and let $e$ be an arc of $\widehat{F}$. Then at least one leaf node of $F$ is reachable from $e$ in $\widehat{F}$.

Let $H$ be an undirected Halin graph $H G\left(k^{\prime}, T\right)$, where $k^{\prime} \geq 3$ and $T$ is a tree such that each internal node has degree $\geq k^{\prime}$. Let $k=\left\lfloor\frac{k^{\prime}}{2}\right\rfloor$; thus, $k^{\prime}=2 k$ or $k^{\prime}=2 k+1$. Let $L$ denote the set of leaf nodes of $T$, and let $C$ denote the cycle of $H$ on $L$. Let $\widehat{H}$ denote a smooth orientation of $H$ that satisfies the conditions in Section 1.3. Let the associated smooth orientation of $T$ be denoted by $\widehat{T}$. Moreover, let $\widehat{C}$ denote the orientation of $C$ given by $\widehat{H}$. In the rest of this section, the term leaf node (non-leaf node) means a leaf node (non-leaf node) of $T$.

The main result of this section follows easily from the next result; a proof is given later. 
Proposition 6 In the directed graph $\widehat{H}$, the set $L$ is $k$-node connected.

Theorem 7 The directed graph $\widehat{H}$ (the specified smooth orientation of a Halin graph) is $k$-node connected.

Proof: Our proof follows easily from Proposition 6 . Let $z$ be any node in $V(H)-L$, that is, $z$ is a non-leaf node of one of the copies of $T$. The smooth orientation has at least $k=\left\lfloor\frac{k^{\prime}}{2}\right\rfloor \operatorname{arcs}$ with tail $z$, since $T$ has $\geq k^{\prime}$ edges incident to $z$. Then, by Fact 5 , there is a $k$-fan from $z$ to $L$, since a leaf node is reachable from each of the arcs outgoing from $z$, and moreover, dipaths that start with distinct arcs outgoing from $z$ have no nodes in common except $z$. Similarly, there is a reverse $k$-fan from $L$ to $z$. Hence, by Proposition $4, \widehat{H}$ is $k$-node connected.

Finally, we give the deferred proof.

Proof of Proposition 6: Consider any two nodes $s, t \in L$. Our goal is to show that $\widehat{H}$ has $k$ openly disjoint $s, t$-dipaths. By Menger's Theorem, this holds iff there exists an $s, t$-dipath in the directed graph obtained from $\widehat{H}$ by removing any set of nodes $Q$ of size $\leq k-1$, where $Q \subseteq V(H)-\{s, t\}$. If either $Q$ is empty or $Q$ consists of non-leaf nodes, then $\widehat{H}-Q$ contains the oriented cycle $\widehat{C}$ which contains an $s, t$-dipath.

Otherwise, $Q$ has at least one leaf node, hence, $k \geq 2$ and $k^{\prime} \geq 4$. Moreover, $Q$ has $\leq k-2$ non-leaf nodes. Then there exists a copy of the smooth orientation $\widehat{T}$ of $T$ that contains no non-leaf node of $Q$, because we have $k^{\prime}-2$ copies of the tree $T$ (including $T$ ), and we have $\geq k-1=\left\lfloor\frac{k^{\prime}-2}{2}\right\rfloor$ copies of $\widehat{T}$. Similarly, there exists a copy of the opposite orientation of $\widehat{T}$, call it $\widetilde{T}$, that contains no non-leaf node of $Q$. (In what follows, we also use $\widehat{T}$ and $\widetilde{T}$ to denote these two copies of $T$.) Let $\widehat{T} \cup \widetilde{T}$ denote the directed graph formed by the union of $\widehat{T}$ and $\widetilde{T}$. We claim that removing any set of nodes $R \subset L$ of size $\leq k-1$ from $\widehat{T} \cup \widetilde{T}$ results in a directed graph that is strongly connected. Clearly, the proposition follows from this claim.

Let us use primes to distinguish between the two copies of a node in the two copies of $T$, e.g., if $v$ is a node in $\widetilde{T}$, then $v^{\prime}$ denotes the copy of $v$ in $\widehat{T}$. (If $v \in L$, then $v^{\prime}=v$.) For a non-leaf node $v$, observe that $\widehat{T} \bigcup \widetilde{T}$ has $k$ openly disjoint $v, v^{\prime}$-dipaths; this holds because $\widehat{T}$ contains a $k$-fan from $v$ to $L$, and $\widetilde{T}$ contains the opposite orientation of this $k$-fan. Hence, $v$ and $v^{\prime}$ are in the same strongly connected component of $\widehat{T} \bigcup \widetilde{T}-R$, because this directed graph has a $v, v^{\prime}$-dipath and a $v^{\prime}, v$-dipath.

Finally, consider a pair of adjacent nodes $v, w$ of $T-R$, and consider any copy of $v$ and any copy of $w$ in $\widehat{T} \cup \widetilde{T}-R$. Observe that $\widehat{T} \cup \widetilde{T}-R$ has a $v, w$-dipath. To see this, assume w.l.o.g. that both $v$ and $w$ are in $\widehat{T}$; if the edge $\{v, w\}$ of $T$ is oriented from $v$ to $w$ in $\widehat{T}$, then we are done; otherwise, note that $\widehat{T} \cup \widetilde{T}-R$ contains a $v, v^{\prime}$-dipath, the arc $\left(v^{\prime}, w^{\prime}\right)$, and a $w^{\prime}, w$-dipath, hence, 
it has a $v, w$-dipath.

It follows that $\widehat{T} \bigcup \widetilde{T}-R$ is strongly connected, because $T-R$ is connected.

The above theorem has an immediate corollary for element connectivity.

Corollary 8 Consider a k-element connected graph $H$ that is obtained from a subdivision of a Halin graph $H G(k, T)$ by choosing the set of terminals to be any subset (of size $\geq 2$ ) of the set of nodes of degree $\geq k$. Then $H$ has a smooth orientation that is strongly $\left\lfloor\frac{k}{2}\right\rfloor$-element connected.

Acknowledgments: We thank a number of colleagues for useful discussions and comments on preliminary drafts that helped us in improving the exposition. In particular we thank Olivier de Gevigney, Penny Haxell, Mohit Singh, David Pritchard, and Andres Ruiz Vargas. The first author acknowledges support by NSERC grant No. OGP0138432. 


\section{References}

[1] A.R.Berg and T.Jordán, "Two-connected orientations of Eulerian graphs," J. Graph Theory, $52(3): 230-242,2006$.

[2] J.A.Bondy and U.S.R.Murty, Graph Theory, Springer, Graduate Texts in Mathematics, Vol. 244, New York, 2008.

[3] A.E.Brouwer and J.H.Koolen, "The vertex-connectivity of a distance-regular graph," European J. Combinatorics, 30:668-673, 2009.

[4] S.Even, "An algorithm for determining whether the connectivity of a graph is at least $k$," SIAM J. Comput. 4(3): 393-396, 1975.

[5] S.Even and R.E.Tarjan, "Network flow and testing graph connectivity," SIAM J. Comput. 4(4): 507-518, 1975.

[6] A.Frank, "Connectivity and network flows," in Handbook of Combinatorics 1:111-177, Elsevier, Amsterdam, 1995.

[7] A.Frank, Connections in Combinatorial Optimization, Oxford Lecture Series in Mathematics and Its Applications, Oxford University Press, Oxford, UK, 2011.

[8] C.Godsil and G.Royle, Algebraic Graph Theory, Graduate Texts in Mathematics, Vol. 207, Springer, New York, 2001.

[9] R.Halin, "Studies on minimally n-connected graphs," in: Combinatorial Mathematics and its Applications, D.J.A.Welsh, ed., Academic Press, London \& New York, 1971, 129-136.

[10] T.Jordán, "On the existence of $k$ edge-disjoint 2-connected spanning subgraphs," J. Comb. Theory, Ser.B, 95(2):257-262, 2005.

[11] Z.Király and Z.Szigeti, "Simultaneous well-balanced orientations of graphs," J. Comb. Theory, Ser.B, 96(5):684-692, 2006.

[12] T.Király and L.C.Lau, "Approximate min-max theorems for Steiner rooted-orientations of graphs and hypergraphs," J. Comb. Theory, Ser.B, 98(6):1233-1252, 2008.

[13] C.St.J.A.Nash-Williams, "On orientations, connectivity, and odd vertex pairings in finite graphs," Canad. J. Math., 12:555-567, 1960.

[14] Projective plane, article in Wikipedia. URL: en.wikipedia.org/wiki/Projective_plane

[15] C.Thomassen, "Configurations in graphs of large minimum degree, connectivity, or chromatic number," Ann. NY Acad. Sci., 555:402-412, 1989. 